\title{
On new Fejér type inequalities for $m$-convex and quasi convex functions
}

\author{
Çetin Yildiz and M. Emin Özdemir
}

Atatürk University, K.K. Education Faculty, Department of Mathematics, 25240, Campus, Erzurum, Turkey

E-mail: cetin@atauni.edu.tr, emos@atauni.edu.tr

\begin{abstract}
In this paper we establish new inequalities of weighted version of Hermite-Hadamard type inequality for functions whose derivatives absolute values are $m-$ convex. Also we obtain some Fejér type inequalities for quasi-convex functions.
\end{abstract}

2010 Mathematics Subject Classification. 26D15. 26D10

Keywords. Fejér inequality, Hermite-Hadamard inequality, $m$-convex functions, quasi-convex functions.

\section{Introduction}

The following double inequality is well known in the literature as Hadamard's inequality:

Let $f: I \subseteq \mathbb{R} \rightarrow \mathbb{R}$ be a convex function defined on an interval $I$ of real numbers, $a, b \in I$ and $a<b$, we have

$$
f\left(\frac{a+b}{2}\right) \leq \frac{1}{b-a} \int_{a}^{b} f(x) d x \leq \frac{f(a)+f(b)}{2} .
$$

Both inequalities hold in the reversed direction if $f$ is concave.

It was first discovered by Hermite in 1881 in the Journal Mathesis (see [10]). The inequality (1.1) was nowhere mentioned in the mathematical literature until 1893. Beckenbach, a leading expert on the theory of convex functions, wrote that inequality (1.1) was proven by Hadamard in 1893 (see [11]). In 1974 Mitrinovič found Hermite's note in Mathesis. That is why, the inequality (1.1) was known as Hermite-Hadamard inequality.

In [1], Fejér established the following Fejér inequality which is the weighted generalization of Hermite-Hadamard inequality:

Theorem 1.1. Let $f: I \rightarrow \mathbb{R}$ be convex on $I$ and let $a, b \in I$ with $a<b$. Then the inequality

$$
f\left(\frac{a+b}{2}\right) \int_{a}^{b} g(x) d x \leq \int_{a}^{b} f(x) g(x) d x \leq \frac{f(a)+f(b)}{2} \int_{a}^{b} g(x) d x
$$

holds, where $g:[a, b] \rightarrow \mathbb{R}$ is nonnegative and symmetric to $\frac{a+b}{2}$.

If $g=1$, then we are talking about the Hermite-Hadamard inequalities. More about those inequalities can be found in a number of papers and monographies. For recent results and generalizations concerning Fejér inequality (1.2) see [2]-[8]. 
Definition 1.2. A function $f:[a, b] \subset \mathbb{R} \rightarrow \mathbb{R}$ is said to be convex if whenever $x, y \in[a, b]$ and $t \in[0,1]$, the following inequality holds:

$$
f(t x+(1-t) y) \leq t f(x)+(1-t) f(y) .
$$

We say that $f$ is concave if $(-f)$ is convex.

This definition has its origins in Jensen's results from [9] and has opened up the most extended, useful and multi-disciplinary domain of mathematics, namely, convex analysis. Convex curves and convex bodies have appeared in mathematical literature since antiquity and there are many important results related to them.

In [12], G. Toader defined $m$-convexity as the following:

Definition 1.3. The function $f:[0, b] \rightarrow \mathbb{R}, b>0$, is said to be $m$-convex, where $m \in[0,1]$, if we have

$$
f(t x+m(1-t) y) \leq t f(x)+m(1-t) f(y)
$$

for all $x, y \in[0, b]$ and $t \in[0,1]$. Denote by $K_{m}(b)$ the set of the $m$-convex functions on $[0, b]$ for which $f(0) \leq 0$.

In [13], Set et al. proved the following inequality of Hermite-Hadamard type for $m$-convex functions.

Theorem 1.4. Let $f: I^{\circ} \subset\left[0, b^{*}\right] \rightarrow \mathbb{R}, b^{*}>0$, be a differentiable mapping on $I^{\circ}, a, b \in I^{\circ}$ with $a<b$. If $\left|f^{\prime}\right|^{q}$ is $m$-convex on $[a, b], q>1$ and $m \in(0,1]$, then the following inequality holds:

$$
\begin{aligned}
& \left|\frac{1}{b-a} \int_{a}^{b} f(x) d x-f\left(\frac{a+b}{2}\right)\right| \\
\leq & \frac{(b-a)}{4}\left\{\left(\left|f^{\prime}(a)\right|^{q}+3 m\left|f^{\prime}\left(\frac{b}{m}\right)\right|^{q}\right)^{\frac{1}{q}}+\left(3\left|f^{\prime}(a)\right|^{q}+m\left|f^{\prime}\left(\frac{b}{m}\right)\right|^{q}\right)^{\frac{1}{q}}\right\} .
\end{aligned}
$$

In [8], Sarıkaya proved the following Lemmas for Fejér type inequalities:

Lemma 1.5. Let $f: I^{\circ} \subset \mathbb{R} \rightarrow \mathbb{R}$ be a differentiable mapping on $I^{\circ}, a, b \in I^{\circ}$ with $a<b$ and $w:[a, b] \rightarrow[0, \infty)$ be a differentiable mapping. If $f^{\prime} \in L[a, b]$, then the following equality holds:

$$
\int_{a}^{b} f(x) w(x) d x-f\left(\frac{a+b}{2}\right) \int_{a}^{b} w(x) d x=(b-a)^{2} \int_{0}^{1} k(t) f^{\prime}(t a+(1-t) b) d t
$$

for each $t \in[0,1]$, where

$$
k(t)=\left\{\begin{array}{cc}
\int_{0}^{t} w(s a+(1-s) b) d s, & t \in\left[0, \frac{1}{2}\right) \\
-\int_{t}^{1} w(s a+(1-s) b) d s, & t \in\left[\frac{1}{2}, 1\right] .
\end{array}\right.
$$


Lemma 1.6. Let $f: I^{\circ} \subset \mathbb{R} \rightarrow \mathbb{R}$ be a differentiable mapping on $I^{\circ}, a, b \in I^{\circ}$ with $a<b$ and $w:[a, b] \rightarrow[0, \infty)$ be a differentiable mapping. If $f^{\prime} \in L[a, b]$, then the following equality holds:

$$
\frac{f(a)+f(b)}{2} \int_{a}^{b} w(x) d x-\int_{a}^{b} f(x) w(x) d x=\frac{(b-a)^{2}}{2} \int_{0}^{1} p(t) f^{\prime}(t a+(1-t) b) d t
$$

for each $t \in[0,1]$, where

$$
p(t)=\int_{t}^{1} w(s a+(1-s) b) d s-\int_{0}^{t} w(s a+(1-s) b) d s .
$$

The aim of this paper is to establish new inequalities of weighted version of Hermite-Hadamard type inequality for functions whose derivatives absolute values are $m$ - convex. Also we obtain some new Fejér type inequalities for quasi-convex functions.

\section{Inequalities for $m$-convex functions}

Theorem 2.1. Let $f: I^{\circ} \subset \mathbb{R} \rightarrow \mathbb{R}$ be a differentiable mapping on $I^{\circ}, a, b \in I^{\circ}$ with $a<b$ and $w:[a, b] \rightarrow[0, \infty)$ be a differentiable mapping. If $\left|f^{\prime}\right|$ is $m$-convex on $[a, b]$ for some fixed $m \in(0,1]$ then the following inequality holds:

$$
\begin{aligned}
& \left|\int_{a}^{b} f(x) w(x) d x-f\left(\frac{a+b}{2}\right) \int_{a}^{b} w(x) d x\right| \\
\leq & \frac{(b-a)^{2}}{6}\left\{\|w\|_{\left[0, \frac{1}{2}\right], \infty}\left(\left|f^{\prime}(a)\right|+2 m\left|f^{\prime}\left(\frac{b}{m}\right)\right|\right)+\|w\|_{\left[\frac{1}{2}, 1\right], \infty}\left(2\left|f^{\prime}(a)\right|+m\left|f^{\prime}\left(\frac{b}{m}\right)\right|\right)\right\} \\
\leq & \frac{(b-a)^{2}}{8}\|w\|_{[0,1], \infty}\left(\left|f^{\prime}(a)\right|+m\left|f^{\prime}\left(\frac{b}{m}\right)\right|\right) .
\end{aligned}
$$

Proof. From Lemma 1.5, using the properties of modulus, we have

$$
\begin{aligned}
&\left|\int_{a}^{b} f(x) w(x) d x-f\left(\frac{a+b}{2}\right) \int_{a}^{b} w(x) d x\right| \\
& \leq \quad(b-a)^{2}\left\{\int_{0}^{\frac{1}{2}}\left|\int_{0}^{t} w(s a+(1-s) b) d s\right|\left|f^{\prime}(t a+(1-t) b)\right| d t\right. \\
&+\left.\int_{\frac{1}{2}}^{1}\left|\int_{t}^{1} w(s a+(1-s) b) d s\right|\left|f^{\prime}(t a+(1-t) b)\right| d t\right\} \\
& \leq \quad(b-a)^{2}\left\{\|w\|_{\left[0, \frac{1}{2}\right], \infty} \int_{0}^{\frac{1}{2}} t\left|f^{\prime}(t a+(1-t) b)\right| d t\right. \\
&\left.+\|w\|_{\left[\frac{1}{2}, 1\right], \infty} \int_{\frac{1}{2}}^{1}(1-t)\left|f^{\prime}(t a+(1-t) b)\right| d t\right\} .
\end{aligned}
$$


Since $\left|f^{\prime}\right|$ is $m$-convex on $[a, b]$, we know that for $t \in[0,1]$

$$
\left|f^{\prime}(t a+(1-t) b)\right|=\left|f^{\prime}\left(t a+m(1-t) \frac{b}{m}\right)\right| \leq t\left|f^{\prime}(a)\right|+m(1-t)\left|f^{\prime}\left(\frac{b}{m}\right)\right|,
$$

hence

$$
\begin{aligned}
\left|\int_{a}^{b} f(x) w(x) d x-f\left(\frac{a+b}{2}\right) \int_{a}^{b} w(x) d x\right| \\
\leq(b-a)^{2}\left\{\|w\|_{\left[0, \frac{1}{2}\right], \infty} \int_{0}^{\frac{1}{2}} t\left[t\left|f^{\prime}(a)\right|+m(1-t)\left|f^{\prime}\left(\frac{b}{m}\right)\right|\right] d t\right. \\
\left.+\|w\|_{\left[\frac{1}{2}, 1\right], \infty} \int_{\frac{1}{2}}^{1}(1-t)\left[t\left|f^{\prime}(a)\right|+m(1-t)\left|f^{\prime}\left(\frac{b}{m}\right)\right|\right] d t\right\} \\
\leq \frac{(b-a)^{2}}{6}\left\{\|w\|_{\left[0, \frac{1}{2}\right], \infty}\left(\left|f^{\prime}(a)\right|+2 m\left|f^{\prime}\left(\frac{b}{m}\right)\right|\right)\right. \\
\left.+\|w\|_{\left[\frac{1}{2}, 1\right], \infty}\left(2\left|f^{\prime}(a)\right|+m\left|f^{\prime}\left(\frac{b}{m}\right)\right|\right)\right\} .
\end{aligned}
$$

Also

$$
\|w\|_{\left[0, \frac{1}{2}\right], \infty} \leq\|w\|_{[0,1], \infty}
$$

and

$$
\|w\|_{\left[\frac{1}{2}, 1\right], \infty} \leq\|w\|_{[0,1], \infty}
$$

by using (2.2), we obtain (2.1). This completes the proof.

Theorem 2.2. Let $f: I^{\circ} \subset \mathbb{R} \rightarrow \mathbb{R}$ be a differentiable mapping on $I^{\circ}, a, b \in I^{\circ}$ with $a<b$ and $w:[a, b] \rightarrow[0, \infty)$ be a differentiable mapping. If $\left|f^{\prime}\right|$ is $m$-convex on $[a, b], q>1$, for some fixed $m \in(0,1]$ then the following inequality holds:

$$
\begin{aligned}
& \left|\int_{a}^{b} f(x) w(x) d x-f\left(\frac{a+b}{2}\right) \int_{a}^{b} w(x) d x\right| \\
& \leq \frac{(b-a)^{2}}{4(p+1)^{1 / p}}\left\{\|w\|_{\left[0, \frac{1}{2}\right], \infty}\left(\frac{\left|f^{\prime}(a)\right|^{q}+3 m\left|f^{\prime}\left(\frac{b}{m}\right)\right|^{q}}{4}\right)^{\frac{1}{q}}\right. \\
& \left.+\|w\|_{\left[\frac{1}{2}, 1\right], \infty}\left(\frac{3\left|f^{\prime}(a)\right|^{q}+m\left|f^{\prime}\left(\frac{b}{m}\right)\right|^{q}}{4}\right)^{\frac{1}{q}}\right\} \\
& \leq \frac{(b-a)^{2}}{4(p+1)^{1 / p}}\|w\|_{[0,1], \infty}\left\{\left(\frac{\left|f^{\prime}(a)\right|^{q}+3 m\left|f^{\prime}\left(\frac{b}{m}\right)\right|^{q}}{4}\right)^{\frac{1}{q}}+\left(\frac{3\left|f^{\prime}(a)\right|^{q}+m\left|f^{\prime}\left(\frac{b}{m}\right)\right|^{q}}{4}\right)^{\frac{1}{q}}\right\} .
\end{aligned}
$$


Proof. Using Lemma 1.5 and Hölder inequality, we obtain

$$
\begin{aligned}
&\left|\int_{a}^{b} f(x) w(x) d x-f\left(\frac{a+b}{2}\right) \int_{a}^{b} w(x) d x\right| \\
& \leq \quad(b-a)^{2}\left\{\int_{0}^{\frac{1}{2}}\left|\int_{0}^{t} w(s a+(1-s) b) d s\right|\left|f^{\prime}(t a+(1-t) b)\right| d t\right. \\
&\left.+\int_{\frac{1}{2}}^{1}\left|\int_{t}^{1} w(s a+(1-s) b) d s\right|\left|f^{\prime}(t a+(1-t) b)\right| d t\right\} \\
& \leq(b-a)^{2}\left\{\left(\int_{0}^{\frac{1}{2}}\left|\int_{0}^{t} w(s a+(1-s) b) d s\right|^{p} d t\right)^{\frac{1}{p}}\left(\int_{0}^{\frac{1}{2}}\left|f^{\prime}(t a+(1-t) b)\right|^{q} d t\right)^{\frac{1}{q}}\right. \\
&\left.+\left(\int_{\frac{1}{2}}^{1}\left|\int_{t}^{1} w(s a+(1-s) b) d s\right|^{p} d t\right)^{\frac{1}{p}}\left(\int_{\frac{1}{2}}^{1}\left|f^{\prime}(t a+(1-t) b)\right|^{q} d t\right)^{\frac{1}{q}}\right\} \\
& \leq(b-a)^{2}\left\{\|w\|_{\left[0, \frac{1}{2}\right], \infty}\left(\int_{0}^{\frac{1}{2}} t^{p} d t\right)^{\frac{1}{p}}\left(\int_{0}^{\frac{1}{2}}\left|f^{\prime}(t a+(1-t) b)\right|^{q} d t\right)^{\frac{1}{q}}\right. \\
&\left.+\|w\|_{\left[\frac{1}{2}, 1\right], \infty}\left(\int_{\frac{1}{2}}^{1}|1-t|^{p} d t\right)^{\frac{1}{p}}\left(\int_{\frac{1}{2}}^{1}\left|f^{\prime}(t a+(1-t) b)\right|^{q} d t\right)^{\frac{1}{q}}\right\}
\end{aligned}
$$

for $\frac{1}{p}+\frac{1}{q}=1$. Since $\left|f^{\prime}\right|^{q}$ is $m$-convex on $[a, b]$, we have

$$
\begin{gathered}
\left|\int_{a}^{b} f(x) w(x) d x-f\left(\frac{a+b}{2}\right) \int_{a}^{b} w(x) d x\right| \\
\leq \frac{(b-a)^{2}}{4(p+1)^{1 / p}}\left\{\|w\|_{\left[0, \frac{1}{2}\right], \infty}\left(\int_{0}^{\frac{1}{2}}\left[t\left|f^{\prime}(a)\right|^{q}+m(1-t)\left|f^{\prime}\left(\frac{b}{m}\right)\right|^{q}\right] d t\right)^{\frac{1}{q}}\right. \\
\left.+\|w\|_{\left[\frac{1}{2}, 1\right], \infty}\left(\int_{\frac{1}{2}}^{1}\left[t\left|f^{\prime}(a)\right|^{q}+m(1-t)\left|f^{\prime}\left(\frac{b}{m}\right)\right|^{q}\right] d t\right)^{\frac{1}{q}}\right\} \\
=\frac{(b-a)^{2}}{4(p+1)^{1 / p}}\left\{\|w\|_{\left[0, \frac{1}{2}\right], \infty}\left(\frac{\left|f^{\prime}(a)\right|^{q}+3 m\left|f^{\prime}\left(\frac{b}{m}\right)\right|^{q}}{4}\right)^{\frac{1}{q}}\right. \\
\left.+\|w\|_{\left[\frac{1}{2}, 1\right], \infty}\left(\frac{3\left|f^{\prime}(a)\right|^{q}+m\left|f^{\prime}\left(\frac{b}{m}\right)\right|^{q}}{4}\right)^{\frac{1}{q}}\right\}
\end{gathered}
$$

Also

$$
\int_{0}^{\frac{1}{2}} t^{p} d t=\int_{\frac{1}{2}}^{1}(1-t)^{p} d t=\frac{1}{2^{p+1}(p+1)}
$$


This completes the proof.

Remark 2.3. Since $\left(\frac{1}{p+1}\right)^{\frac{1}{p}}<1$ and $\frac{1}{4^{1 / q}}<1$, if we choose $w(x)=1$ in Theorem 2.2 , we obtain the inequalties (1.3).

Theorem 2.4. Let $f: I^{\circ} \subset \mathbb{R} \rightarrow \mathbb{R}$ be a differentiable mapping on $I^{\circ}, a, b \in I^{\circ}$ with $a<b$ and $w:[a, b] \rightarrow[0, \infty)$ be a differentiable mapping. If $\left|f^{\prime}\right|$ is $m-$ convex on $[a, b]$ for some fixed $m \in(0,1]$ then the following inequality holds:

$$
\begin{aligned}
& \left|\frac{f(a)+f(b)}{2} \int_{a}^{b} w(x) d x-\int_{a}^{b} f(x) w(x) d x\right| \\
\leq & \frac{(b-a)^{2}}{4}\|w\|_{[0,1], \infty} \min \left\{\left|f^{\prime}(a)\right|+m\left|f^{\prime}\left(\frac{b}{m}\right)\right|, m\left|f^{\prime}\left(\frac{a}{m}\right)\right|+\left|f^{\prime}(b)\right|\right\} .
\end{aligned}
$$

Proof. Let $x \in[a, b]$. Using Lemma 1.6, we have

$$
\begin{aligned}
& \left|\frac{f(a)+f(b)}{2} \int_{a}^{b} w(x) d x-\int_{a}^{b} f(x) w(x) d x\right| \\
\leq & \frac{(b-a)^{2}}{2}\left\{\int_{0}^{1}\left|\int_{0}^{t} w(s a+(1-s) b) d s\right|\left|f^{\prime}(t a+(1-t) b)\right| d t\right. \\
& \left.+\int_{0}^{1}\left|\int_{t}^{1} w(s a+(1-s) b) d s\right|\left|f^{\prime}(t a+(1-t) b)\right| d t\right\} \\
\leq & \frac{(b-a)^{2}}{2}\|w\|_{[0,1], \infty}\left\{\int_{0}^{1} t\left|f^{\prime}(t a+(1-t) b)\right| d t+\int_{0}^{1}|1-t|\left|f^{\prime}(t a+(1-t) b)\right| d t\right\} .
\end{aligned}
$$

Since $\left|f^{\prime}\right|$ is $m$-convex on $[a, b]$, we obtain

$$
\begin{aligned}
&\left|\frac{f(a)+f(b)}{2} \int_{a}^{b} w(x) d x-\int_{a}^{b} f(x) w(x) d x\right| \\
& \leq \frac{(b-a)^{2}}{2}\|w\|_{[0,1], \infty}\left\{\int_{0}^{1} t\left[t\left|f^{\prime}(a)\right|+m(1-t)\left|f^{\prime}\left(\frac{b}{m}\right)\right|\right] d t\right. \\
&\left.+\int_{0}^{1}(1-t)\left[t\left|f^{\prime}(a)\right|+m(1-t)\left|f^{\prime}\left(\frac{b}{m}\right)\right|\right] d t\right\} \\
&=\frac{(b-a)^{2}}{4}\|w\|_{[0,1], \infty}\left\{\left|f^{\prime}(a)\right|+m\left|f^{\prime}\left(\frac{b}{m}\right)\right|\right\} .
\end{aligned}
$$

Analogously we have

$$
\left|\frac{f(a)+f(b)}{2} \int_{a}^{b} w(x) d x-\int_{a}^{b} f(x) w(x) d x\right| \leq \frac{(b-a)^{2}}{4}\|w\|_{[0,1], \infty}\left\{m\left|f^{\prime}\left(\frac{a}{m}\right)\right|+\left|f^{\prime}(b)\right|\right\},
$$

which completes the proof. 
Theorem 2.5. Let $f: I^{\circ} \subset \mathbb{R} \rightarrow \mathbb{R}$ be a differentiable mapping on $I^{\circ}, a, b \in I^{\circ}$ with $a<b$ and $w:[a, b] \rightarrow[0, \infty)$ be a differentiable mapping. If $\left|f^{\prime}\right|^{q}$ is $m$-convex on $[a, b], q>1$, for some fixed $m \in(0,1]$ then the following inequality holds:

$$
\begin{aligned}
& \left|\frac{f(a)+f(b)}{2} \int_{a}^{b} w(x) d x-\int_{a}^{b} f(x) w(x) d x\right| \\
\leq & \frac{(b-a)^{2}}{(p+1)^{\frac{1}{p}}}\|w\|_{[0,1], \infty} \min \left\{\left[\frac{\left|f^{\prime}(a)\right|^{q}+m\left|f^{\prime}\left(\frac{b}{m}\right)\right|^{q}}{2}\right]^{\frac{1}{q}},\left[\frac{m\left|f^{\prime}\left(\frac{a}{m}\right)\right|^{q}+\left|f^{\prime}(b)\right|^{q}}{2}\right]^{\frac{1}{q}}\right\}
\end{aligned}
$$

where $\frac{1}{p}+\frac{1}{q}=1$.

Proof. Using Lemma 1.6, Hölder's inequality and the $m$-convexity of $\left|f^{\prime}\right|^{q}$, for $\frac{1}{p}+\frac{1}{q}=1$, it follows that

$$
\begin{aligned}
& \left|\frac{f(a)+f(b)}{2} \int_{a}^{b} w(x) d x-\int_{a}^{b} f(x) w(x) d x\right| \\
\leq & \frac{(b-a)^{2}}{2}\left\{\left(\int_{0}^{1}\left|\int_{0}^{t} w(s a+(1-s) b) d s\right|^{p} d t\right)^{\frac{1}{p}}\left(\int_{0}^{1}\left|f^{\prime}(t a+(1-t) b)\right|^{q} d t\right)^{\frac{1}{q}}\right. \\
& \left.+\left(\int_{0}^{1}\left|\int_{t}^{1} w(s a+(1-s) b) d s\right|^{p} d t\right)^{\frac{1}{p}}\left(\int_{0}^{1}\left|f^{\prime}(t a+(1-t) b)\right|^{q} d t\right)^{\frac{1}{q}}\right\} \\
\leq & \frac{(b-a)^{2}}{2}\|w\|_{[0,1], \infty}\left\{\left(\int_{0}^{1} t^{p} d t\right)^{\frac{1}{p}}\left(\int_{0}^{1}\left[t\left|f^{\prime}(a)\right|^{q}+m(1-t)\left|f^{\prime}\left(\frac{b}{m}\right)\right|^{q}\right] d t\right)^{\frac{1}{q}}\right. \\
& \left.+\left(\int_{0}^{1}(1-t)^{p} d t\right)^{\frac{1}{p}}\left(\int_{0}^{1}\left[t\left|f^{\prime}(a)\right|^{q}+m(1-t)\left|f^{\prime}\left(\frac{b}{m}\right)\right|^{q}\right] d t\right)^{\frac{1}{q}}\right\} \\
= & \frac{(b-a)^{2}}{(p+1)^{\frac{1}{p}}}\|w\|_{[0,1], \infty}\left[\frac{\left|f^{\prime}(a)\right|^{q}+m\left|f^{\prime}\left(\frac{b}{m}\right)\right|^{q}}{2}\right]^{\frac{1}{q}}
\end{aligned}
$$

and analogously

$$
\left|\frac{f(a)+f(b)}{2} \int_{a}^{b} w(x) d x-\int_{a}^{b} f(x) w(x) d x\right| \leq \frac{(b-a)^{2}}{(p+1)^{\frac{1}{p}}}\|w\|_{[0,1], \infty}\left[\frac{m\left|f^{\prime}\left(\frac{a}{m}\right)\right|^{q}+\left|f^{\prime}(b)\right|^{q}}{2}\right]^{\frac{1}{q}}
$$

which completes the proof.

\section{Inequalities for quasi-convex functions}

Theorem 3.1. Let $f:[0, \infty) \rightarrow \mathbb{R}$ be a quasi-convex function, $a, b \in[0, \infty)$ with $a<b$ and $g:[a, b] \rightarrow \mathbb{R}$ be nonnegative, integrable and symetric about $\frac{a+b}{2}$. Then

$$
\int_{a}^{b} f(x) g(x) d x \leq \max \{f(a), f(b)\} \int_{a}^{b} g(x) d x .
$$


Proof. Since $f$ is quasi-convex and $g$ is nonnegative, integrable and symetric about $\frac{a+b}{2}$, we have

$$
\begin{aligned}
\int_{a}^{b} f(x) g(x) d x & =\frac{1}{2}\left[\int_{a}^{b} f(x) g(x) d x+\int_{a}^{b} f(a+b-x) g(a+b-x) d x\right] \\
& =\frac{1}{2}\left\{\int_{a}^{b}[f(x)+f(a+b-x)] g(x) d x\right\} \\
& =\frac{1}{2}\left\{\int_{a}^{b}\left[f\left(\frac{b-x}{b-a} a+\frac{x-a}{b-a} b\right)+f\left(\frac{x-a}{b-a} a+\frac{b-x}{b-a} b\right)\right] g(x) d x\right\} \\
& \leq \frac{1}{2}\left\{\int_{a}^{b}[\max \{f(a), f(b)\}+\max \{f(a), f(b)\}] g(x) d x\right\} \\
& =\max \{f(a), f(b)\} \int_{a}^{b} g(x) d x .
\end{aligned}
$$

This completes the proof.

\section{References}

[1] L. Fejér, Uberdie Fourierreihen, II, Math. Naturwise. Anz Ungar. Akad., Wiss, 24 (1906), 369-390, (in Hungarian).

[2] F. Qi and Z.-L. Yang, Generalizations and refinements of Hermite-Hadamard's inequality, The Rocky Mountain J. of Math., 35(2005), 235-251.

[3] S.-H. Wu, On the weighted generalization of the Hermite-Hadamard inequality and its applications, The Rocky Mountain J. of Math., 39(2009), 1741-1749.

[4] K.-L. Tseng, G.-S. Yang and K.-C. Hsu, Some inequalities for differentiable mappings and applications to Fejér inequality and weighted trapezoidal formula, Taiwanese J. of Math., $15(4)(2011), 1737-1747$.

[5] K.-L. Tseng, S.R. Hwang, and S.S. Dragomir, On some new inequaties of Hermite-HadamardFejér type involving convex functions, Demons. Math., 40(1)(2007).

[6] K.-L. Tseng, S.R. Hwang, and S.S. Dragomir, Fejér-Type Inequalities (I), Journal of Inequalities and Applications, 2010, 2010:531976.

[7] M. Bombardelli, S. Varošanec, Properties of h-convex functions related to the HermiteHadamard-Fejér inequaliries, Comp. Math. App., 58(2009), 1869-1877.

[8] M.Z. Sarıkaya, On new Hermite Hadamard Fejér type integral inequalities, Stud. Univ. BabeşBolyai Math. 57(3)(2012), 377-386.

[9] J. L. W. V. Jensen, On konvexe funktioner og uligheder mellem middlvaerdier, Nyt. Tidsskr. Math. B., 16, 49-69, 1905. 
[10] D.S. Mitrinovič and I.B. Lackovič, Hermite and convexity, Aequat. Math. 28(1985), 229-232.

[11] E.F. Beckenbach, Convex functions, Bull. Amer. Math. Soc., 54(1948), 439-460.

[12] G. Toader, Some generalizations of the convexity, Proceedings of The Colloquium On Approximation and Optimization, Univ. Cluj-Napoca, Cluj-Napoca, 1984, 329-338.

[13] E. Set, M.E. Özdemir and M.Z. Sarıkaya, Inequalities of Hermite-Hadamard's type for functions whose derivatives absolute values are m-convex, AIP Conferences Proceeding, 1309, 861, (2010). 